\title{
Data Prediction of Deflection BASIN EVOLUTION OF ASPHALT PAVEMENT STRUCTURE BASED ON Multi-LEVEL NeURAL NETWORK
}

\author{
Shaosheng $\mathrm{Xu}^{1}$, Jinde $\mathrm{Cao}^{2}$ and $\mathrm{Xiangnan} \mathrm{Liu}^{2}$ \\ ${ }^{1}$ School of Automation, Southeast University, Nanjing, China \\ ${ }^{2}$ School of Mathematics, Southeast University, Nanjing, China
}

\begin{abstract}
Aiming at reducing the high cost of test data collection of deflection basins in the structural design of asphalt pavement and shortening the long test time of new structures, this paper innovatively designs a structure coding network based on traditional neural networks to map the pavement structure to an abstract space. Therefore, the generalization ability of the neural network structure is improved, and a new multi-level neural network model is formed to predict the evolution data of the deflection basin of the untested structure. By testing the experimental data of RIOHTRACK, the network structure predicts the deflection basin data of untested pavement structure, of which the average prediction error is less than $5 \%$.
\end{abstract}

\section{KEYWORDS}

multi-level neural network, Encoding converter, structural of asphalt pavement, deflection basins, RIOHTRACK

\section{INTRODUCTION}

In the past 20 years, Europe and the United States have established the concept of long-life pavement and permanent pavement. Long-life pavement refers to a pavement where the foundation or pavement base layer will not be significantly aged under the condition of correct pavement maintenance [1]. Most of traditional pavements are semi-rigid base asphalt pavements, which have the advantages of good stability and convenient materials. But there are also disadvantages of poor durability and high maintenance costs. Traditional asphalt pavement has structural damage, mainly manifested as fatigue cracking and permanent deformation, which has a short service life and the dismantling and reconstruction have brought huge economic losses. In order to reduce maintenance and other costs in the future, it will be more cost-effective to extend the design life of roads in places with heavy traffic to at least 40 years without the need to strengthen the structure [2].

Many existing literatures analyse the fatigue of pavement structure through mechanical tests and study the corresponding factors that affect pavement mechanics. Yu et al. carried out four-point bending fatigue tests in constant strain and constant stress modes and established the laboratory fatigue prediction models in these two cases. In addition, the transfer function of loading mode is established to realize the fatigue life conversion. Moreover, the laboratory constant strain prediction model was combined with the loading mode transfer function to establish an asphalt pavement fatigue crack prediction model [3]. Jiang et al. evaluated the strength and fatigue David C. Wyld et al. (Eds): BIBC, AISO, EDTECH - 2020 
performance of asphalt mixtures by semi-circular bending strength test and fatigue test [4]. Wang et al. took several base asphalts and modified asphalts as the research objects and simulated the short-term and long-term aging of asphalt by RTFO and PAV respectively [5]. Gschwendt and Ivan introduced a program of pavement management system based on degradation model, and estimated the repair time of asphalt pavement. Using the theory of pavement mechanics, the stress and strain on the two pavement model layers are calculated [6]. Islam et al. used the existing Per Road and AASHTOW are pavement ME Design software to re-examine the design of the existing four permanent pavement sections to investigate the correctness of the assumptions [7]. Assogba et al. studied the mechanical parameter distributions of three new semirigid pavement structures with typical functions and structural requirements which are specially designed to deal with a variety of damages of semi-rigid pavements [8]. Yang et al. proposed a reinforced slow-cracking stress absorbing layer and compared it with the ordinary SBS modified asphalt stress absorbing layer. Besides, the dynamic response laws of the two stress absorbing layers under different conditions are analysed by the strain data of the two stress absorbing layers under different loads, speeds and temperatures [9].

With the development of machine learning, the application of artificial neural networks has grown tremendously in several fields of engineering, such as road conditions and performance prediction, road pressure prediction, and structural road system evaluation [10]. Gu et al. applied artificial neural networks to predict the response of any given material and structural characteristics to geo-reinforced pavement. Furthermore, the results are applied to the method of improving material performance in pavement design, so as to consider the influence of reinforcement on pavement design under any traffic load and weather conditions [11]. Tapkin et al. proposed a neural network model using the physical properties of the standard Marshall sample to predict the Marshall test results of polypropylene (PP) modified asphalt mixture, and obtained the explicit expressions of stability, flow rate and Marshall quotient [12]. Qadir developed an artificial neural network (ANN) model to predict the bending stiffness and rut depth of the reinforced asphalt pavement using design parameters from simple laboratory procedures of Marshall and rut depth tests [13].

RIOHTRACK was utilized in 2017 to a trial loading test to collect and study the evolutionary law of multi-use performance under the conditions of the full-life service cycle of nonlinear road structures and materials, and to verify and improve the design methods of road structures and materials. Under different load levels, 19 kinds of structures' deflection basins with different stiffness levels have been regularly tested and data collected, and their changes have been analysed [14]. As an important indicator reflecting the bearing capacity of asphalt pavement structures, deflection has always been the focus of highway builders and scientific researcher [1517]. This paper uses the data collected from the RIOHTRACK ring road to predict the deflection basin of the road by establishing a multi-level neural network model. The main contribution of this paper is to establish a multi-level neural network model, which mainly includes three parts: encoding converter, coupler and interpreter. The encoding converter is used to convert the original data of structure to high-dimensional coded data, and the coded data is used as an influencing factor to predict the deflection basin data of the road surface. Through the existing experimental data, the performance of untested pavement structures can be analysed and predicted, which reduces the cost and time of experiments on new structures. While, the coupler checks whether the coded data are appropriate in the high-dimensional space. In other words, it is to make sure that the topology of the coded data is reasonable than which of the data of structure. The interpreter is the component that generate the prediction of the deflection basin data finally. The organization of this paper is as follows. In the second section, a further analysis is given for the problems. After that, the overall framework of the definition and model involved in the paper is given. Subsequently, the multi-level neural network model established in this paper is 
introduced in detail, and the experimental results are given. At the same time, it is compared with other methods. The conclusion is given at the end of the paper.

\section{Problem Analysis}

As mentioned before, the data in this paper was collected from RIOHTRACK, which is a 2039meter-long full-scale test track. Trial loading tests are implemented in RIOHTRACK. It is obviously that the cost of time, labour and finance of such a test are very huge. For the deflection basin of untested pavement structure, even if an abstract physical model is established to simulate the deflection basin data, it is quite difficult to calculate. The research in this paper is based on 19 kinds of experimental pavement data of structure and corresponding other data including temperature, load, number of standard axle load and deflection basin. It is expected to directly predict the performance of the new pavement structure composed of the structural materials involved in the existing pavement without paving the new test road so as to save the pavement research cycle and costs.

In order to achieve the above goals, this paper establishes a new multi-level neural network model consisting of encoding converter, coupler and interpreter. Using the known structure to train the model, the prediction of the test data of the new structure is completed.

\section{DEFINITION AND FRAMEWORK}

This paper mainly predicts the pavement deflection basin by extracting and encoding the structural features of pavements and combining with other features. The different structures are related to each other to form a triple represented by $\mathcal{G}=(V, E, X)$, where $G=(V, E)$ constitutes a graph; $V=\left\{v_{i}\right\}_{i=1,2, \ldots, n}$ represents the set of nodes in the graph ,that is the set of different pavement structures; $E=\left\{e_{i j}\right\}$ is the set of edges between the nodes; $X=\left\{x_{1}, x_{2}, \ldots, x_{n}\right\}$ is the characteristic of nodes, where $x_{i} \in \mathbb{R}^{m}$ is a real valued vector. The characteristic of the node in this paper is the deflection basin performance of the pavement structure. In essence, E represents the relationship between different structures, which can be determined by the pavement structure and the deflection basin, that is $E(V, X)$. A method $f^{*}$ is expected to be found to encode the structure through training the model which satisfies

$$
E\left(V, f^{*}(V)\right) \leq E(V, f(V))
$$

Where $f$ represents any encoding method. 


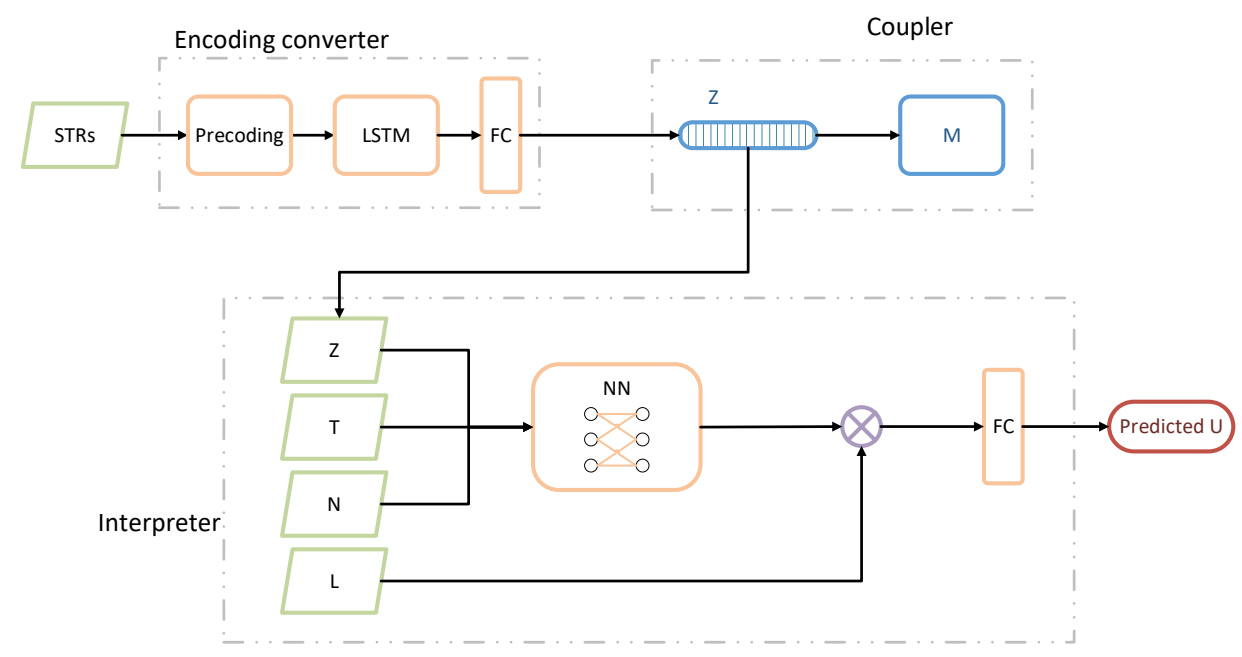

Figure 1. The framework of the model

The overall framework of the model is shown in the Figure 1, which consists of three components: encoding converter, coupler and interpreter. The encoding converter converts the original pavement data into an array through pre-encoding and then encodes further through a Long Short-Term Memory (LSTM) network [18] and a linear layer. The coupler is mainly used to reconstruct the graph and train the network. The interpreter takes the learned coding vector and other factors that affect the deflection basin as input to realize the prediction of deflection basin.

\section{Model ANd Methodology}

In this section, a multi-level neural network model is designed in details. First, the data of structure pass the encoding converter and the coupler to learn the potential encoding vector. Then, the learned encoding vector is used as an explanatory factor to predict the deflection basin with the interpreter.

\subsection{Encoding converter}

The encoding converter mainly includes two processes. To begin with, original data of structure was pre-encoded. The original data of structure contains two parts of information including the structural thickness of each layer and the structural material. As shown in TABLE I, where MAWA refers to the modified asphalt waterproof adhesive layer.

Table 1. The original data of structure.

\begin{tabular}{|c|c|c|c|c|c|}
\hline & STR1 & STR2 & STR3 & STR4 & STR5 \\
\hline Layer1 & 4cm SBS-SAC13 & 4cm SBS-SAC13 & $\begin{array}{l}\text { 4cm SBS- } \\
\text { SAC13 }\end{array}$ & $\begin{array}{l}\text { 4cm SBS- } \\
\text { SAC13 }\end{array}$ & $\begin{array}{l}\text { 4cm SBS- } \\
\text { SAC13 }\end{array}$ \\
\hline Layer2 & MAWA Tpye1 & MAWA Tpye1 & MAWA Tpye1 & MAWA Tpye1 & MAWA Tpye1 \\
\hline Layer3 & $8 \mathrm{~cm} \mathrm{~A} 30-\mathrm{AC} 20$ & 8cm A30-AC20 & $8 \mathrm{~cm} \mathrm{~A} 30-\mathrm{AC} 20$ & $6 \mathrm{~cm} \mathrm{A30-AC20}$ & $6 \mathrm{~cm} \mathrm{A30-AC20}$ \\
\hline Layer4 & MAWA Tpye2 & MAWA Tpye2 & MAWA Tpye2 & clay particles & clay particles \\
\hline Layer5 & $20 \mathrm{~cm}$ CBG-A & $20 \mathrm{~cm}$ CBG-A & $20 \mathrm{~cm} \mathrm{CBG-A}$ & $2 \mathrm{~cm}$ SBS-AC10 & $2 \mathrm{~cm} \mathrm{SBS-AC10}$ \\
\hline Layer6 & 20cm CBG-A & 20cm CBG-A & 20cm CBG-A & $\begin{array}{l}\text { Layer of clay } \\
\text { particles }\end{array}$ & $\begin{array}{l}\text { Layer of clay } \\
\text { particles }\end{array}$ \\
\hline Layer7 & $20 \mathrm{~cm} \mathrm{CS}$ & $20 \mathrm{~cm} \mathrm{CS}$ & $20 \mathrm{~cm} \mathrm{~GB}$ & $24 \mathrm{~cm} \mathrm{LCC}$ & $24 \mathrm{~cm} \mathrm{CC}$ \\
\hline Layer8 & $20 \mathrm{~cm} \mathrm{CS}$ & \multicolumn{2}{|c|}{ None } & 20cm CBG-A & $20 \mathrm{~cm}$ CBG-A \\
\hline Layer9 & \multicolumn{3}{|c|}{ None } & $20 \mathrm{~cm} \mathrm{CS}$ & $20 \mathrm{~cm} \mathrm{CS}$ \\
\hline AC layers & $12 \mathrm{~cm}$ & $12 \mathrm{~cm}$ & $12 \mathrm{~cm}$ & $12 \mathrm{~cm}$ & $12 \mathrm{~cm}$ \\
\hline
\end{tabular}


Let $S$ denote the set of all materials involved in the pavement structures in $V$, and number $s_{j}$ as 1 to $k$ respectively. Therefore, the material thickness and the serial number of the material of each layer together constitute the precoding matrix $\left\{P_{i}\right\}_{i=1,2, \ldots, n}$, where $P_{i} \in \mathbb{R}^{l \times 2}$ and $l$ is determined by the number of structural material layers.

Next, the further encoding is completed through a LSTM network (see Figure 2.) and a linear layer. The space of the precoding matrix is defined as the coding space $\mathbb{F}$. After the precoding matrix is converted by LSTM, the coding expression on the abstract space $\mathbb{H}$ is obtained and the mapping from the coding space to the abstract space is realized and the result is denoted by $\widetilde{z_{t}}$, which is followed by the normalization process $z=W_{z} \cdot \widetilde{z_{t}}+b_{z}$. Let $Z=\left\{z_{1}, z_{2}, \ldots, z_{n}\right\}$ represents the coding vector for all the structures.

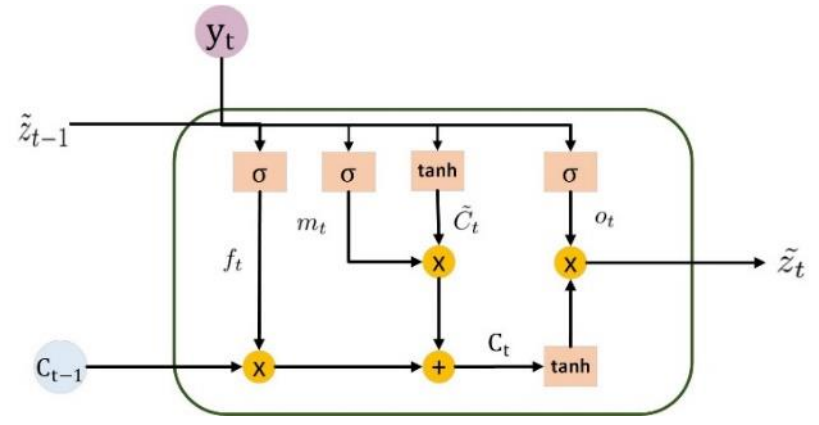

Figure 2. The structure of LSTM

It should be noted that the serial number of structural materials in the precoding process can be in any order, without considering the similarity and correlation between the materials. Because the similarity and association of the structure can be reflected by the output of LSTM as long as the serial number of the same material is the same.

\subsection{Coupler}

Coupler is mainly used to train the aforementioned LSTM network through reconstruction. A variety of existing decoder scan achieve structural reconstruction [18]. In this paper, characteristics are used for reconstruction. Firstly, transform the characteristic (i.e. deflection basin) as follows to obtain the matrix $\mathrm{M}$,

$$
M=\operatorname{DFT}\left(I X-(I X)^{T}\right)
$$

where DFT is discrete Fourier transform, $I$ is an array whose elements are all one and matrix $M$ is defined as attribute adjacency matrix. The coding sequence $Z$ is used to obtain the reconstructed matrix $M^{\prime}$ as follows,

$$
M^{\prime}=I Z-(I Z)^{T}
$$

where $W$ and $b$ are the weight matrix and bias respectively. The reconstruction loss function is defined as $L_{s}=\operatorname{loss}_{1}\left(M^{\prime}, M\right)$.

The parameters of LSTM network are adjusted by minimizing the loss function. 


\subsection{Interpreter}

The ultimate goal of this paper is to predict deflection basins of untested pavements. The existing results show that the deflection basin has a great relationship with temperature, load, the number of standard axle load, and obviously has a close relationship with the pavement structure. The features of the pavement structure have been extracted above, and the coding structure sequence can reflect the structural features and connections of different pavements. Through a simple linear regression analysis of the data, it can be seen that there is a clear linear relationship between the deflection basin and the load. Therefore, a semi-display neural network interpreter as shown in Figure 1 is designed. The input of the interpreter is the coding sequence $Z$, the number of standard axle load N, the temperature $T$ and the load $L$. In this paper, the accumulative number of standard axle load is adopted as $N$.

First, input $Z, N, T$ into a neural network to get the compressed feature $g=g(Z, N, T)$. The compression feature $\mathrm{g}$ and the load $\mathrm{L}$ are subjected to the Hadamard product, and the dimension compression is performed through the fully connected layer to obtain the predicted deflection basin $\widehat{U}=W_{h}(g * L)+b_{h}$, where * is Hadamard product. It can be seen that the black box part of the network is only for the extraction of the compression feature $g$, and the relationship between the load and the deflection basin is fully displayed by the parameters of the fully connected layer. The final forecast error is defined as $L_{P}=\operatorname{loss}_{2}(\widehat{U}, U)$.

The parameters in the interpreter are updated by back propagation of errors to achieve the minimum error loss.

\section{EXPERIMENTS}

In this section, the data used in the experiment is introduced and the evaluation criteria of the model is explained, so as the parameter settings. And the results of the experiment are analysed after the results' display. The whole experiment is implemented by Python/Pytorch.

\subsection{Data and Setting of Parameters}

The data used in this paper comes from China's first full-scale pavement test ring road named RIOHTRACK. As shown in the Figure 3, the ring road is an oval closed curve divided into two sections of straight and cylinder. A total of 19 kinds of pavement structures have been designed (In view of the large amount of data loss in STR19, the data of this experimental structure is abandoned), including the flexible, semi-rigid and rigid base structure, as well as different asphalt structure layers and material combination modes [14].

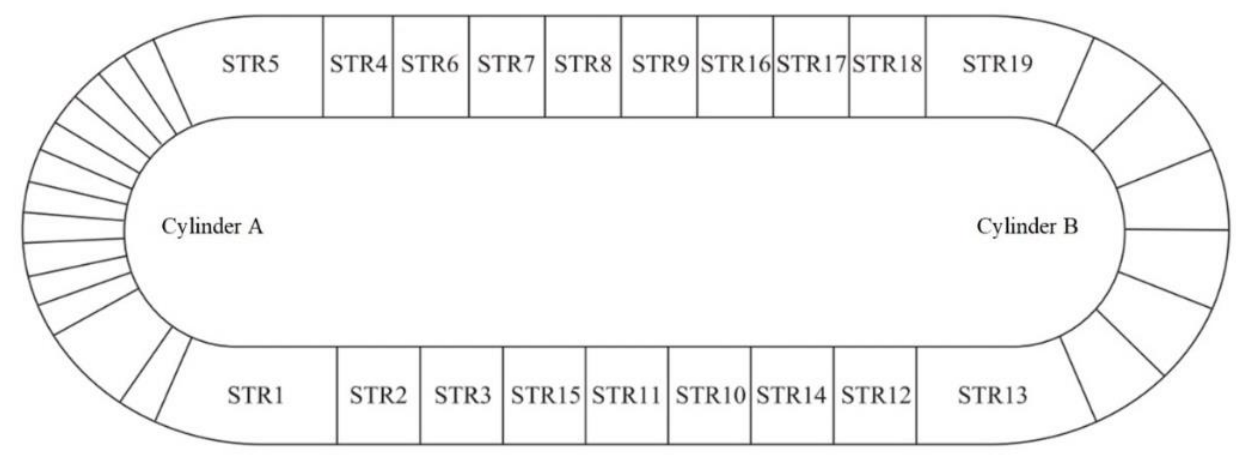

Figure 3. Schematic diagram of RIOHTRACK test section 
The 19 kinds of pavement structures are divided into a training set and a test set. The training set contains 16 structures, $D=\{$ STR1, STR2, STR3, STR4, STR5, STR6, STR7, STR8, STR9, STR10, STR11, STR12, STR13, STR14, STR15, STR17\} and the remaining two structures constitute the test set $V=\{$ STR16, STR18 $\}$. By deleting the missing data, there are 256 data for each pavement structure. Therefore, there are $256 \times 16=4096$ data in training set and $256 \times$ $2=512$ data in test set when predicting and the mean square errors (MSE) are used as the evaluation criteria to estimate the effectiveness of the model. The loss functions of both components are MSE loss and the learning rates are set to 0.02 . The number of training iterations is 3000 and 1000 respectively. Due to the lack of the training data, the complexity of coding layer is reduced, such as using single-layer LSTM and single-layer FC, to prevent overfitting.

\subsection{Experiment Results}

The Figure 4 shows the prediction results of the two structures of STR16 and STR18 in the test set. It can be seen that the overall performance of the model proposed in this paper is well, but at some peaks, it looks like a little bad. The average MSE of all the data in the test set is 0.0455 , which also shows that the overall prediction is effective. It can be seen from Figure 4 that the predicted value of the upper peak position is often significantly higher, because the horizontal axis in Figure 4 represents time, and the position of the upper peak is in the summer period, so it can be concluded that the prediction of this model has obvious upper deviations under high temperature. The specific analysis is given in the discussion section.

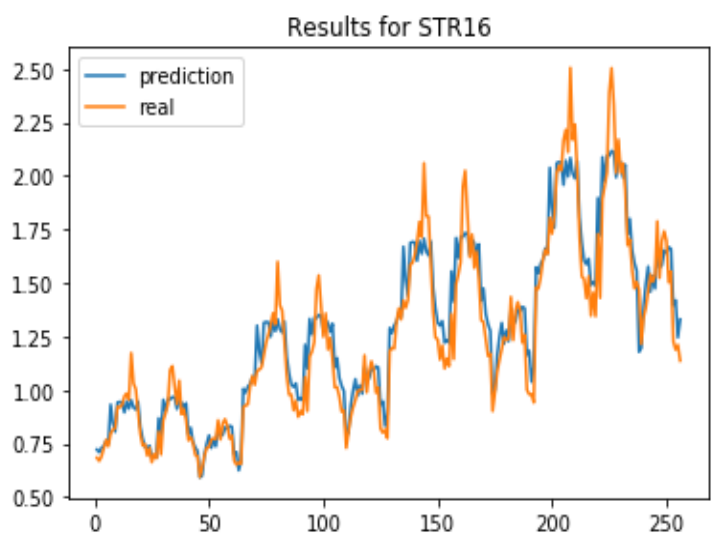

(a)

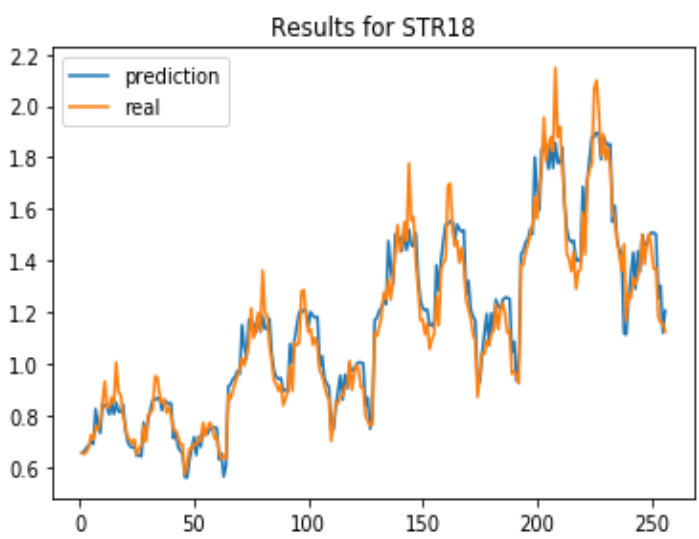

(b)

Figure 4(a) Result of the multi-level neural network for STR16 and Figure 4(b) Result of the multi-level neural network for STR18

In order to verify the important role of the encoding converter in prediction, as a comparison, the precoding structure vector is used directly as a prediction factor and other factors to predict the deflection basin through the interpreter. The distribution of training data and test data is the same as before. As shown in Figure 5, it can be seen that the prediction results are worse than our model intuitively and the MSE of the normal network is over 79.3 in the test set. 


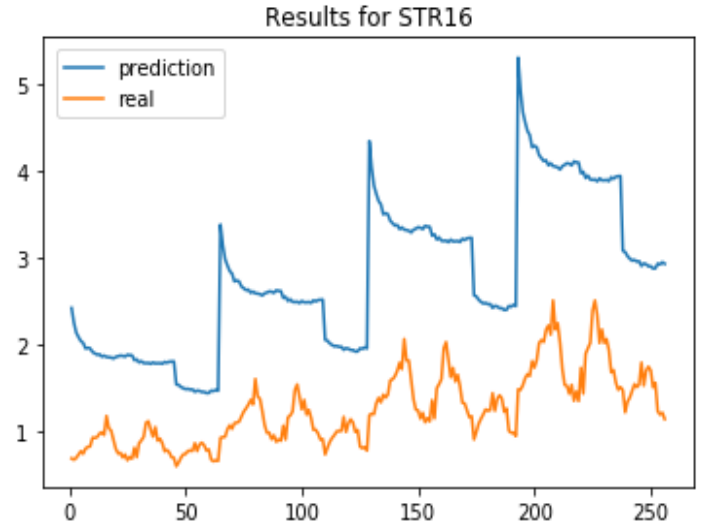

(a)

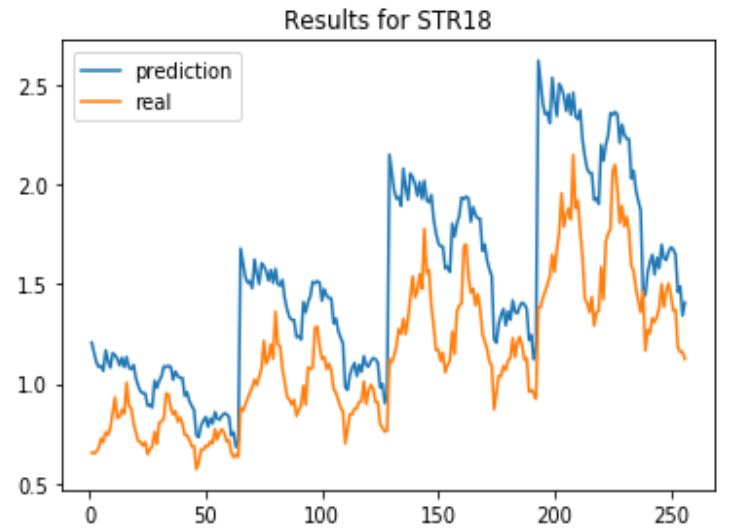

(b)

Figure 5(a) Result of the normal neural network for STR16 and Figure 5(b) Result of the normal neural network for STR18

\section{DISCUSSION}

In preliminary analysis, the reason for the larger prediction error of the normal neural network is that the characteristics of the space in which the data of structure is located are significantly different from the characteristics of the space in which the physical quantity is being tested. The former is a logical expression space on the intuitive level with a spatial order structure, while the latter is a physical space on the dynamic level with a temporal order structure. However, it is difficult to analyse the time sequence of the multi-level structural space, so the nonlinear fitting function of the normal neural network is difficult to play its due role.

It is may be noticed that the data of structure and the one of experimental test variables have different dimensions, and considered that the different dimensions cause the larger deviation of the normal neural network prediction results. Here a different opinion is. Because even in the interpreter module, the dimensions of the input variables are not the same, but the multilayer neural network proposed in this paper has a less error (the average error is less than 5\%). At present, a large number of neural network models can input variables of different dimensions at the same time and achieve good results, which means that neural network models can overcome the problems caused by different dimensions to a certain extent.

In the problem dealt with in this paper, the data of structure is a vector in an abstract space (human intuitive cognitive space) compared to variables such as temperature, load, etc., which is like the direct transcoding of textual expressions. The encoding module and coupler module proposed in this paper are similar with the function of Word $2 \mathrm{Vec}$ in the natural language processing. After the coding is completed, the topological structure between codes can better express the topological relationship between them at the time of testing, so it can directly reflect the physical meaning represented by a structure, that is, the original intuitive cognitive space is mapped to the physical space, and the consistency of the two spaces is guaranteed, so that a better calculation result can be obtained.

The reason for the upper deviation of the upper peak position forecast is not yet conclusive. In view of the poor interpretation ability of the neural network itself, it is difficult to analyse it theoretically. What is certain at present is that the period when the predictions show a significant upward shift occurs in the summer, when is during the high temperature period of a year. Therefore, it can be concluded that high temperature may be an important cause of prediction errors. It is precisely because of this inference that this issue can be considered from the 
following two aspects. First, the objective pavement structure has a sudden change in response to temperature switch. That is, near some high temperature, the material mechanical properties of the overall structure change suddenly. Some local mechanical laws suddenly change from approximate linear laws to obvious nonlinear laws. An important reason which supports this guess is that asphalt is a non-Newtonian fluid, so whether its multilayer composite materials will also have similar properties is a question, and which may lead the peak value to be significantly lower than the predicted value. Of course, if one wants to verify this conjecture, a large amount of experimental data is needed. The related experiments are currently underway. Second, in terms of the model receiving temperature data, the proportion of high temperature data is very small. That is, for temperature data, the data sample lacks balance, which causes large errors in high temperature training to be flattened as a whole during the back-propagation process. For this reason, a novel interpreter module is under research at the same time.

\section{Conclusions}

In this paper, a multi-level neural network model has been proposed that can encode data of structure and predict the deflection basin of asphalt pavements. It can be seen from the experimental results that structure coding is effective and important. The method proposed in this paper can use the existing data to directly predict the deflection basin of untested pavement, saving the time and capital cost of repeated experiments and the mean prediction loss is under $5 \%$. However, this method relies on a large amount of historical experimental data. Therefore, besides the present research mentioned in the discussion section above, the model development based on small sample training is still in progress.

\section{ACKNOWLEDGEMENTS}

Thanks to the Highway Science Research Institute of the Ministry of Transport, they have provided enough experimental data on asphalt pavements of various structures.

\section{REFERENCES}

[1] E. A. P. Association et al., (2007) "Long-life asphalt pavements-technical version".

[2] M. Nunn, A. Brown, D. Weston, and J. Nicholls, (1997) "Design of long-life flexible pavements for heavy traffic", TRL Limited.

[3] J. Yu, B.-W. Tsai, X. Zhang, and C. Monismith, (2012) "Development of asphalt pavement fatigue cracking prediction model based on loading mode transfer function", Road Materials and Pavement Design, Vol. 13, No. 3, pp. 501-517.

[4] J. Jiang, F. Ni, Q. Dong, F. Wu, and Y. Dai, (2018) "Research on the fatigue equation of asphalt mixtures based on actual stress ratio using semi-circular bending test", Construction and Building Materials, Vol. 158, pp. 996-1002.

[5] Z. Wang and F. Ye, (2020) "Experimental investigation on aging characteristics of asphalt based on rheological properties", Construction and Building Materials, Vol. 231, p. 117158.

[6] I. Gschwendt, (2018) "Extending the service life of pavements", Slovak Journal of Civil Engineering, Vol. 26, No. 1, pp. 25-32.

[7] S. Islam, A. Sufian, M. Hossain, R. Miller, and C. Leibrock, (2020) "Mechanistic-empirical design of perpetual pavement," Road Materials and Pavement Design, Vol. 21, No. 5, pp. 1224-1237.

[8] O. C. Assogba, Y. Tan, X. Zhou, C. Zhang, and J. N. Anato, (2020) "Numerical investigation of the mechanical response of semi-rigid base asphalt pavement under traffic load and nonlinear temperature gradient effect", Construction and Building Materials, Vol. 235, p. 117406.

[9] S. Yang, P. Li, M. Guo, S. Liao, and H. Wu, (2020) "Study on dynamic load monitoring of an enhanced stress absorption layer", Frontiers in Materials, Vol. 7, p. 148.

[10] H. Ceylan, M. B. Bayrak, and K. Gopalakrishnan, (2014) "Neural networks applications in pavement engineering: A recent survey", International Journal of Pavement Research \& Technology, Vol. 7, No. 6, pp. 434-444. 
[11] F. Gu, X. Luo, Y. Zhang, Y. Chen, R. Luo, and R. L. Lytton, (2018) "Prediction of geogridreinforced flexible pavement performance using artificial neural network approach", Road Materials \& Pavement Design, Vol. 19, No. 5-6, pp. 1147-1163.

[12] S. Tapkin, A. Cevik, and U. Usar, (2010) "Prediction of marshal test results for polypropylene modified dense bituminous mixtures using neural networks", Expert Systems with Applications, Vol. 37, No. 6, pp. 4660-4670.

[13] A. Qadir, U. Gazder, and K. U. N. Choudhary, (2020) "Artificial neural network models for performance design of asphalt pavements reinforced with geosynthetics", Transportation Research Record, Vol. 4, p. 0361198120924387.

[14] X. D. Wang, (2017) "Design of pavement structure and material for full-scale test track", Journal of Highway and Transportation Research and Development, Vol. 34, No. 6, pp. 30-37.

[15] X. Wang, (2015) "Discussion of asphalt pavement deflection indicator," Journal of Highway and Transportation Research and Development, Vol. 32, No. 1, pp. 1-12.

[16] J. Liao, et al. (2019) "A Correction Model for the Continuous Deflection Measurement of Pavements Under Dynamic Loads”, IEEE Access, Vol. 7, pp. 154770-154785.

[17] C. Wu, H. Wang, et al., (2020) "Asphalt pavement modulus back calculation using surface deflections under moving loads", Computer-Aided Civil and Infrastructure Engineering, doi: $10.1111 /$ mice. 12624

[18] C. Wang, S. Pan, R. Hu, et al., (2019) "Attributed graph clustering: A deep attentional embedding approach," arXiv preprint arXiv:1906.06532.

\section{AUTHORS}

Shaosheng Xu received the B.S. degree from University of Jinan, Jinan, China, in 2011 and M.S. degrees from Anhui Normal University, Wuhu, China, in 2016. He is currently pursuing the Ph.D. degree with the School of Automation, Southeast University, Nanjing, China. His current research interests include stochastic optimal control, multi-agent system, multi-robot system and artificial intelligence.

Jinde Cao (F'16) received the B.S. degree from Anhui Normal University, Wuhu, China, the M.S. degree from Yunnan University, Kunming, China, and the Ph.D. degree from Sichuan University, Chengdu, China, all in mathematics /applied mathematics, in 1986, 1989, and 1998, respectively. He is an Endowed Chair Professor, the Dean of the School of Mathematics, the Director of the Jiangsu Provincial Key Laboratory of Networked Collective Intelligence of China and the Director of the Research Center for Complex Systems and Network Sciences at Southeast University. Prof. Cao was a recipient of the National Innovation Award of China, Obada Prize and the Highly Cited Researcher Award in Engineering, Computer Science, and Mathematics by Thomson Reuters/Clarivate Analytics. He is a fellow of IEEE, a member of the Academy of Europe, a member of the European Academy of Sciences and Arts, a fellow of Pakistan Academy of Sciences, and an IASCYS academician.

Xiangnan Liu received the B.Sc. degree in statistics from Southeast University, Nanjing, in 2019.She is currently a postgraduate in Southeast University. Her research interest is machine learning.

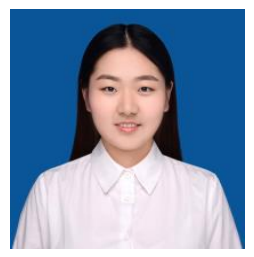

(C) 2020 By AIRCC Publishing Corporation. This article is published under the Creative Commons Attribution (CC BY) license. 